\title{
Seguridad y Protección Radiológica en Procedimientos Imagenológicos Dentales
}

\author{
Safety and Radiological Protection in Dental Imaging Procedures
}

\author{
Carlos Ubeda'; 'Diego Nocetti' \& Marco Aragón²
}

UBEDA, C.; NOCETTI, D. \& ARAGÓN, M. Seguridad y protección radiológica en procedimientos imagenológicos dentales. Int. J. Odontostomat., 12(3):246-251, 2018.

RESUMEN: Los procedimientos de imagenología dental son el tipo más frecuente de estudio radiológico y aportan información esencial para apoyar la evaluación, diagnóstico y tratamiento de patologías y/o alteraciones dentomaxilofaciales. La presente revisión narrativa, muestra los procedimientos de radiología dental, define conceptos asociados a la protección radiológica en éstos y entrega una guía con recomendaciones para mejorar la seguridad y protección radiológica del paciente y del operador, en esta clase de procedimientos imagenológicos. Comprender que bajo el actual sistema de protección radiológica, es el Odontólogo quién debe justificar la realización o no de un tipo de procedimiento radiológico, teniendo presente los costos económicos y sobre todo los costos en términos de dosis para el paciente.

PALABRAS CLAVE: radiología dental, seguridad y protección radiológica.

\section{INTRODUCCIÓN}

La radiología o imagenología dental, consiste en el uso de los rayos $X$ (clasificada como radiación ionizante) emitidos por diversos equipos específicos para el diagnóstico y seguimiento de los estudios odontológicos, donde nos interesa evaluar la pieza dentaria completa o su ausencia, sus posiciones con respecto a las demás, así como el estudio y diagnóstico de quistes, tumores, asimetrías y la valoración del desarroIlo del complejo maxilofacial (Ruiz Manzano, 2005). La elección del tipo de exploración más adecuado en radiología dental depende de diversos factores: cobertura y resolución de cada técnica, exposición relativa del paciente y enfermedades detectables con cada examen.

De acuerdo con el Comité Científico de Naciones Unidas sobre los Efectos de la Radiación Atómica (United Nations Scientific Committee on the Effects of Atomic Radiations, 2016), las exposiciones médicas a radiaciones ionizantes representan actualmente la principal fuente artificial de irradiación a la población mundial (United Nations Scientific Committee on the Effects of Atomic Radiations). Los procedimientos de radiología dental son el tipo más frecuente de estudio radiológico, aunque las dosis individuales son pequeñas.
Junto con lo anterior, es sabido que las radiaciones ionizantes tienen un potencial de producir efectos adversos en la salud de los seres vivos. Es así como los efectos de las radiaciones ionizantes se clasifican en dos categorías principales, basadas en el mecanismo biológico subyacente: las que son el resultado de la muerte celular se denominan reacciones tisulares o efectos deterministas. Tales efectos incluyen eritema cutáneo, pérdida de cabello, cataratas, infertilidad, enfermedad vascular, efectos hematopoyéticos y gastroenterológicos. Aquellos dentro de la segunda categoría, que son el resultado de mutaciones celulares, se conocen como efectos estocásticos e incluyen el cáncer y los efectos genéticos (International Commission on Radiation Protection, 2007).

Por tanto, los objetivos de la presente revisión narrativa fueron: realizar la revisión de los procedimientos de radiología dental, definir conceptos asociados a la protección radiológica en éstos y entregar una guía con recomendaciones para mejorar la seguridad y protección radiológica del paciente y del operador, en esta clase de procedimientos imagenológicos.

\footnotetext{
${ }^{1}$ Departamento de Tecnología Médica, Facultad de Ciencias de la Salud, Universidad de Tarapacá, Arica, Chile.
}

${ }^{2}$ Ortodoncista. Clínica Arisa, Arica, Chile. 


\section{DESARROLLO}

Tipos de procedimientos. Las distintas técnicas se agrupan básicamente en dos clases de radiografías: intraorales y extraorales.

Radiografías intraorales. Los procedimientos intraorales comprenden las radiografías aisladas de los dientes y se realizan colocando la película o detector radiográfico dentro de la boca del paciente. Las categorías de exámenes intraorales son tres: proyecciones periapicales, proyecciones de aleta mordida o Bite-wing y proyecciones oclusales. En las radiografías periapicales se observa el diente completo y las estructuras que lo rodean. Las coronas de los dientes superiores e inferiores y las crestas alveolares adyacentes en una sola imagen son mostradas en las radiografías de aleta mordida, mientras que las radiografías oclusales permiten visualizar un área de hueso y dientes mayor que en las imágenes periapicales (Fig. 1) (Cabrero Fraile, 2004).
Radiografías extraorales. Las radiografías extraorales son exámenes de la región orofacial que se emplean para el estudio de áreas no cubiertas por las proyecciones intraorales o para visualizar el cráneo y las estructuras faciales. En los exámenes radiológicos extraorales la película o detector se colocan fuera de la boca.

En primer lugar encontramos a las radiografías craneales que requieren un posicionamiento adecuado del paciente mediante la utilización de marcas de posición esqueléticas y un sistema que permita colocar correctamente la cabeza (cefalostato). Las líneas de referencia utilizadas son el plano de Frankfort, que conecta el borde superior del conducto auditivo externo con el borde infraorbitario, y el plano cantomeatal definido por el centro del conducto auditivo externo y el ángulo externo del ojo (forma un ángulo de $10^{\circ} \mathrm{con}$ el plano anterior). Las radiografías craneales se realizan a partir de tres proyecciones estándares (Fig. 2): posteroanterior, lateral y axial (Cabrero Fraile).
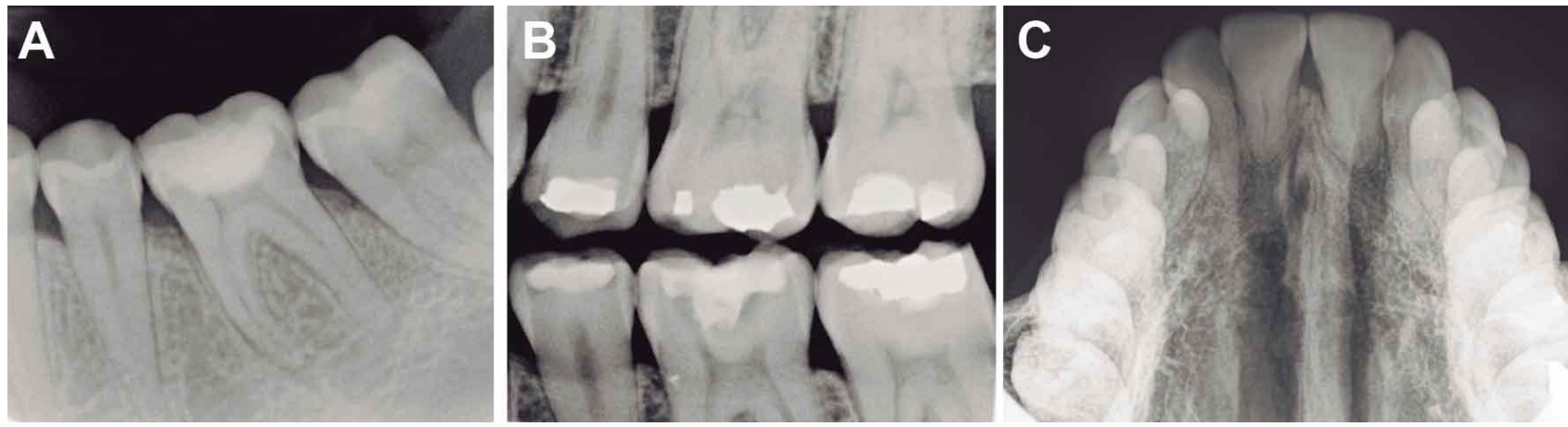

Fig. 1. Imágenes radiográficas de las proyecciones dentales A. periapical, B. de aleta mordida y C. oclusal.
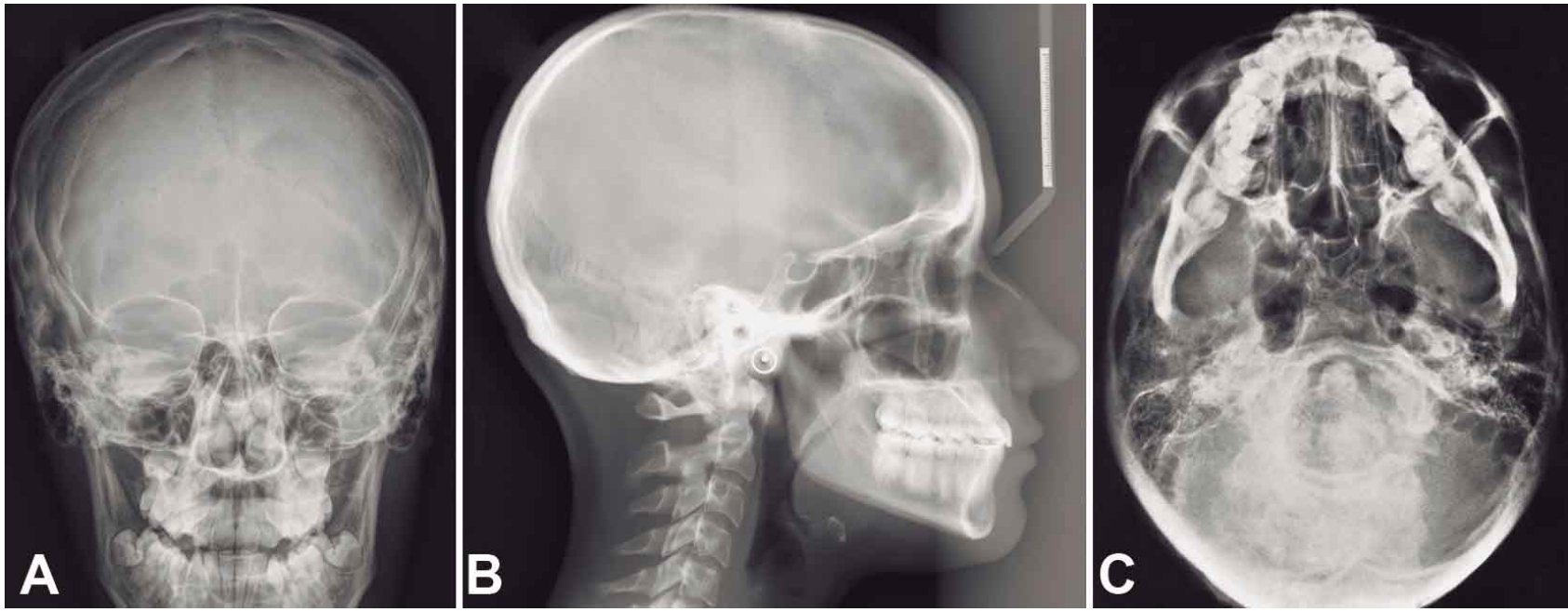

Fig. 2. Imágenes radiográficas de las proyecciones craneales A. posteroanterior, B. lateral y C. axial. 
En segundo lugar tenemos la radiografía dental panorámica u ortopantomografía (Fig. 3), que constituye la fuente básica de información general en radiología odontológica proporcionando una visión global del tercio medio e inferior de la cara que incluye ambos maxilares, ambas articulaciones temporomandibulares y senos maxilares (White \& Pharoah, 2004). Es especialmente útil para mostrar las etapas en el desarrollo dental o anomalías en ella, además, permite evaluar lesiones óseas inflamatorias o traumáticas y son cómodas para los pacientes en comparación con las proyecciones intraorales (Vandenberghe et al., 2010).

Por otra parte encontramos la Tomografía Computarizada (TC) que consiste en una exploración de cráneo donde el plano de corte es paralelo al reborde alveolar, los cortes son generalmente de $1 \mathrm{~mm}$ de grosor y espaciados cada $1 \mathrm{~mm}$. Se realizan por separado de maxilar inferior y de maxilar superior, y un programa específico de reconstrucción nos muestra imágenes transversales y panorámicas múltiples de los maxilares. Es de gran utilidad en implantología ya que proporciona medidas anatómicas muy exactas (Fig. 4).

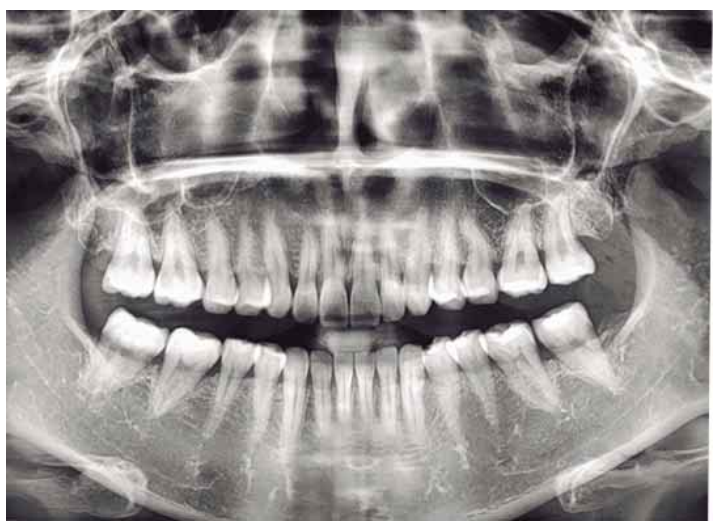

Fig. 3. Radiografía dental panorámica u ortopantomografía.

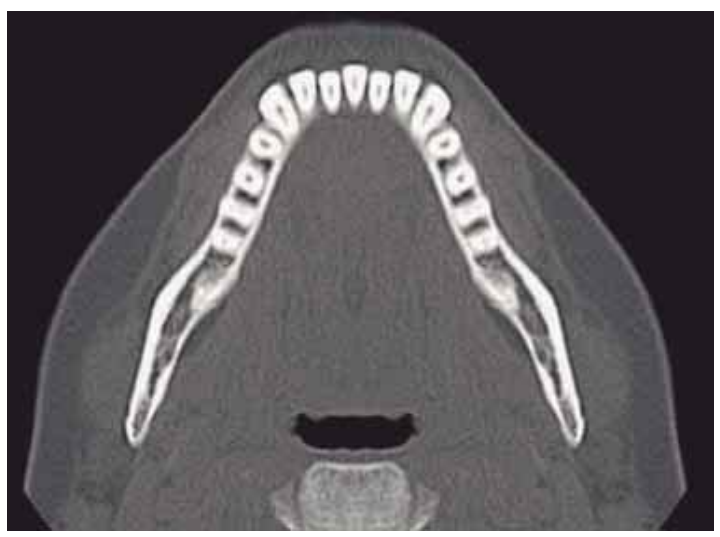

Fig. 4. Tomografía computarizada dental.
Posteriormente, la TC evolucionó hacia la Tomografía computarizada multidetector (MDCT), donde la matriz de detectores (compuesta por más de una fila de éstos) y el tubo de rayos $\mathrm{X}$ giran en conjunto. Las adquisiciones helicoidales se realizan mediante la tecnología de anillo deslizante (Cabrero Fraile).

Finalmente, encontramos la Tomografía computada de haz cónico (CBCT), que es una modalidad de tomografía computarizada donde el haz de rayos $\mathrm{X}$ posee forma cónica, irradiando una matriz bidimensional de detectores, para formar la imagen. Su uso se ha incrementado en diversas especialidades de la odontología debido a las imágenes 3D que permite obtener, aumentando con ello la precisión en el diagnóstico y mejorando los abordajes terapéuticos (Fig. 5) (Vandenberghe et al.).

Definición de conceptos (European Commission, 2004; International Commission on Radiation Units and Measurements, 2005; Ruiz Manzano; Vañó et al., 2017):

Rayos X: Energía electromagnética en forma de fotones, capaz de arrancar electrones de los átomos, pudiendo con ello, provocar cambios en la materia.

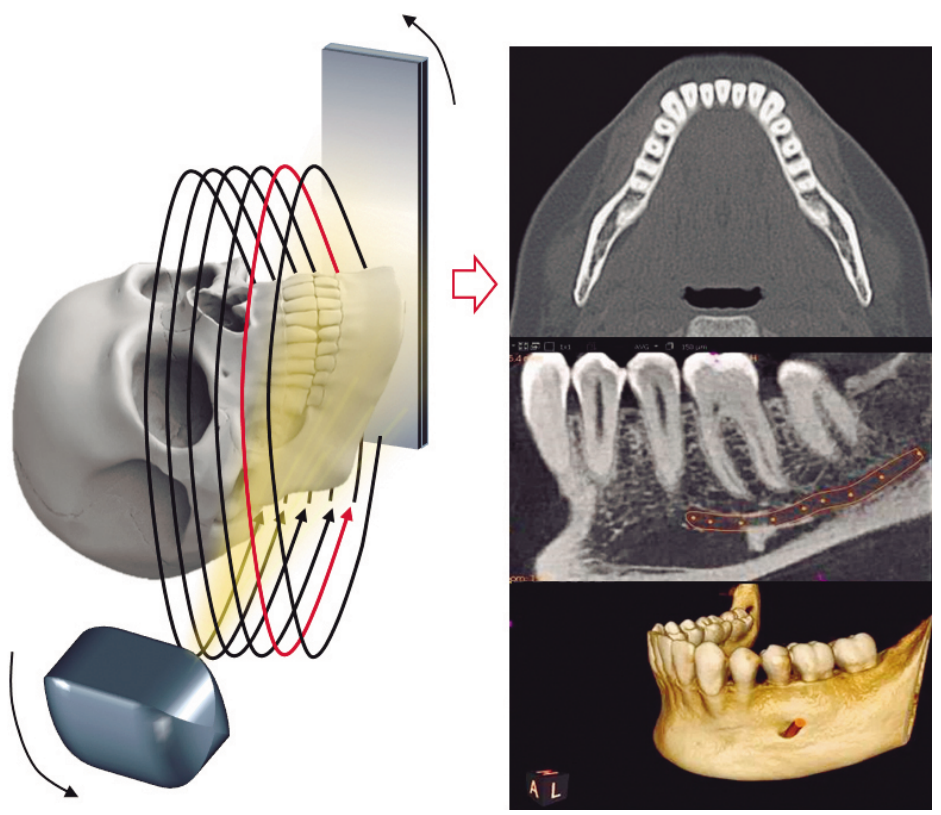

Fig. 5. Tomografía computada de haz cónico.

Dosis absorbida: Magnitud dosimétrica empleada para describir el promedio de la energía depositada en determinada masa de materia. Se expresa en J/kg o en su unidad especial el Gray (Gy). 
Dosis equivalente: Es una magnitud de protección radiológica derivada de la dosis absorbida en tejidos específicos de un individuo, considerando los efectos del tipo y de la energía de la radiación ionizante. Se expresa en Sievert (Sv).

Dosis efectiva: Es una magnitud de protección radiológica que incorpora a la dosis equivalente el efecto de la irradiación en tejidos específicos de un individuo, considerando la probabilidad de cada uno de éstos de sufrir un detrimento de tipo estocástico. Se expresa en Sievert (Sv). También se utiliza esta magnitud para comparar diferentes técnicas imagenológicas (Tabla I).

Tabla I. Comparación de dosis efectiva en exámenes imagenológicos (Mattler et al., 2008).

\begin{tabular}{lc}
\hline Examen & $\begin{array}{c}\text { Dosis efectiva } \\
\text { media (mSv) }\end{array}$ \\
\hline Radiografía de tórax postero-anterior & 0,02 \\
Radiografía de columna lumbar frontal & 1,50 \\
Mamografía & 0,40 \\
TC de cráneo & 2,00 \\
TC de abdomen & 8,00 \\
Angiografía corona ria & 16,0 \\
Radiografía intraoral & 0,01 \\
Ortopantomografía & 0,01 \\
TC dental & 0,20 \\
\hline
\end{tabular}

Producto dosis área: Magnitud que corresponde al producto de la dosis por el área del campo de radiación. Se utiliza preferentemente para la dosimetría de pacientes y el establecimiento de Niveles Referenciales Diagnósticos en radiografías intraorales y algunas extraorales (proyecciones de cráneo y ortopantomografía). Se expresa en unidades de $\mathrm{Gy} \cdot \mathrm{cm}^{2}$.

Niveles Referenciales Diagnósticos (NRDs): Los NRDs son una forma de nivel de investigación utilizada como herramienta para ayudar a optimizar la protección radiológica en la exposición médica de pacientes en procedimientos de diagnóstico e

Tabla II. NRD propuestos para Reino Unido en procedimientos dentales (FGDP, 2013).

\begin{tabular}{ll}
\hline Examen & NRD Reino Unido \\
\hline Intraoral (molar, adulto) & $1,7 \mathrm{mGy}$ \\
Ortopantomografía (adulto) & $93 \mathrm{mGy} \cdot \mathrm{cm}^{2}$ \\
Ortopantomografía (niño) & $67 \mathrm{mGy} \cdot \mathrm{cm}^{2}$ \\
Radiografía lateral cefalométrica (adulto) & $40 \mathrm{mGy} \cdot \mathrm{cm}^{2}$ \\
Radiografía lateral cefalométrica (niño) & $25 \mathrm{mGy} \cdot \mathrm{cm}^{2}$ \\
CBCT (implante en primer molar superior, adulto) & $250 \mathrm{mGy} \cdot \mathrm{cm}^{2}$ \\
\hline
\end{tabular}

intervencionismo. Se utiliza para indicar si, en condiciones de rutina, la cantidad de radiación utilizada para determinado procedimiento es inusualmente alta o baja. La Tabla II, muestra los NRDs propuestos en el Reino Unido.

\section{Protección radiológica:}

Sistema de protección radiológica. La protección radiológica es una disciplina cuyo objetivo es prevenir la ocurrencia de efectos determinísticos y limitar el riesgo de efectos estocásticos a niveles considerados aceptables. Para cumplir con este objetivo, contamos con el Sistema de protección radiológica, el cual se sustenta en tres principios o pilares (International Commission on Radiation Protection, 2007):

a. Justificación de las prácticas: Según este pilar todo examen que utilice radiaciones ionizantes, debería ser prescrito por un médico que ha evaluado previamente todas las alternativas diagnósticas, determinando que el beneficio neto para el paciente es superior que el potencial riesgo al que se expone éste.

b. Optimización: Este principio hace referencia a que en cada procedimiento debe emplearse una dosis tan baja como sea razonablemente alcanzable, considerando factores económicos y sociales, para generar un examen de calidad diagnóstica. La principal herramienta utilizada aquí es el establecimiento de los NRDs.

c. Limitación de la dosis: Es un pilar de la protección radiológica que establece límites de dosis equivalente personal a los trabajadores que se desempeñan en el servicio radiológico (trabajadores ocupacionalmente expuestos (TOE)) y a sujetos que se encuentren en el servicio radiológico (público general). Para la limitación de la ocurrencia de efectos estocásticos, se emplea como magnitud física la Dosis Efectiva, en Chile el máximo anual es 50 mSv (5 rem) para los TOE y $5 \mathrm{mSv}$ para el PG (Decreto Supremo 133, 1984). Según la International Commission on Radiation Protection (ICRP), no es apropiado aplicar límites de dosis a la exposición médica de los pacientes, porque en ellos el pilar de la justificación respalda la irradiación y su cuantía, dado el mayor beneficio para éste (International Commission on Radiation Protection, 2007).

1.1. Recomendaciones genéricas (Wold Dental Federation, 2014; Isaacson et al., 2015). 
a. Receptores de imagen: Siempre que sea posible es recomendable el uso de películas radiográficas o receptores digitales de mayor velocidad o sensibilidad, dado que requieren una menor dosis para la formación de la imagen.

b. Valores de exposición (kVp, mA y tiempo de exposición): Con el objetivo de mejorar el contraste radiográfico y minimizar la dosis en la profundidad de los tejidos del paciente, se sugieren tensiones entre 60 y $70 \mathrm{kVp}$. Asimismo, debería regularse la intensidad de corriente de filamento $(\mathrm{mA})$ y/o el tiempo de exposición, según las características del paciente irradiado.

c. Control automático de exposición (CAE): Debería emplearse esta opción en los equipos radiográficos que la dispongan, no obstante, es esencial una apropiada calibración del sistema, para minimizar la repetición de exámenes por error en los valores de exposición. En equipos que no cuenten con CAE es apropiado contar con una lista de valores de exposición sugeridos.

d. Campo de irradiación: Para radiografías intraorales se sugieren campos de irradiación inferiores a 6 o $7 \mathrm{~cm}$ de diámetro en la piel del paciente. Para otros exámenes, la colimación debería restringirse al área de interés.

e.Visualización de imágenes: Para asegurar la apropiada visualización de las imágenes radiográficas, debería emplearse negatoscopios o monitores de computador apropiadamente calibrados y sometidos a controles de calidad periódicos.

f. Controles de calidad: Deberían ejecutarse controles de calidad periódicos al equipamiento para asegurar su adecuado funcionamiento y uso seguro, abarcando aspectos como: el equipo radiográfico dental, cuarto oscuro, procesadora, material radiográfico (películas, sensores digitales), cassettes, entre otros.

g. Medidas de protección radiológica: Todo individuo que se encuentre en la sala de procedimientos con excepción del paciente, debería situarse al menos a dos metros de la fuente de radiación, detrás de barreras de protección siempre que sea posible, las cuales deben tener una composición apropiada, en función de la cantidad de procedimientos realizados semanalmente y de los valores de exposición empleados típicamente en los procedimientos (levantamiento radiométrico). Adicionalmente, tanto trabajadores, como acompañantes y pacientes, deben utilizar medidas de protección radiológica físicas como protecto- res tiroideos y/o delantales plomados, estos últimos deberán aplicarse según lo permita el examen.

h. Educación y entrenamiento: Quienes manipulen equipos radiográficos dentales deben contar con la respectiva certificación, así como con una adecuada capacitación para el uso apropiado del sistema. Finalmente es fundamental la formación continua del personal para mejorar las prácticas en radiodiagnóstico y protección radiológica.

\section{DISCUSIÓN}

En nuestro país, las normativas que regulan el uso de las radiaciones ionizantes en aplicaciones médicas y dentales (Decreto Supremo 133; Decreto Supremo 3, 1985), están absolutamente desactualizadas de las más recientes recomendaciones realizadas por el Organismo Internacional de Energía Atómica (OIEA) en el año 2014 (International Atomic Energy Agency, 2014). Esta situación y sus alcances han sido comentadas en artículos previos por parte del equipo de investigación (Ubeda et al., 2007; Ubeda et al., 2013; Miranda et al., 2014; Ubeda et al., 2015). Sin embargo, Chile ha participado de iniciativas promovidas por el propio OIEA y existe un documento denominado Manual de Protección Radiológica y de buenas prácticas en radiología dentomaxilofacial, el cual es un interesante texto sobre estas temáticas, pero al parecer no ha tenido la difusión necesaria en todo el país (Ministerio de Salud - Instituto de Salud Pública, 2008).

De acuerdo con lo recomendado por la Comisión Internacional de Protección Radiológica, los Odontólogos deberían recibir capacitación en procedimientos de radiología y protección radiológica como parte de su educación (Vañó et al., 2009) y por medio de cursos de actualización en forma permanente (Vañó et al., 2017). Asimismo, resulta fundamental que la capacitación considere a todo el personal del servicio, especialmente a los tecnólogos médicos y asistentes dentales, debido a que son quienes tienen mayor contacto con el paciente en el contexto del examen de radiología dental. En ese sentido, debe asegurarse la comprensión de aspectos básicos como: qué son los rayos $\mathrm{X}$, las diferentes definiciones sobre la dosis (absorbida, equivalente, efectiva, etc.) y la relevancia de los NRDs, como principal herramienta en la optimización de las dosis o niveles de radiación a los pacientes. Entender que bajo el actual sistema de protección radiológico, es el Odontólogo quién debe justificar la realización o no 
de un tipo de procedimiento radiológico, teniendo presente los costos económicos y sobre todo los costos en términos de dosis para el paciente.

UBEDA, C.; NOCETTI, D. \& ARAGÓN, M. Safety and radiological protection in dental imaging procedures. Int. J. Odontostomat., 12(3):246-251, 2018.

ABSTRACT: Dental imaging procedures are the most frequent type of radiological study and provide essential information to support the evaluation, diagnosis and treatment of pathologies and / or dentomaxillofacial alterations. The present narrative review shows dental radiology procedures, defines concepts associated with radiological protection in these and provides a guide with recommendations to improve the safety and radiological protection of the patient and the operator in this kind of imaging procedures. Understanding that under the current system of radiological protection, it is the dentist who must confirm the realization or not, of a type of radiological procedure, keeping in mind the financial costs, and above all, consequences to the patient in terms of dose used in the procedure.

KEY WORDS: dental radiology, safety and radiation protection.

\section{REFERENCIAS BIBLIOGRÁFICAS}

Cabrero Fraile, F. J. Imagen Radiológica: Principios Físicos e Instrumentación. Masson, Elsevier, 2004.

Decreto Supremo №133 (DS 133). Reglamento sobre Autorizaciones para Instalaciones Radioactivas o Equipos Generadores de Radiaciones lonizantes, Personal que se Desempeña en Ellas, u Opere tales Equipos y otras Actividades Afines. Santiago de Chile, Gobierno de Chile, 1984.

Decreto Supremo №3 (DS 3). Reglamento de Protección Radiológica de Instalaciones Radiactivas. Santiago de Chile, Gobierno de Chile, 1985

European Commission. Radiation Protection 136. European Guidelines on Radiation Protection in Dental Radiology. The Safe Use of Radiographs in Dental Practice. Issue $N^{0} 136$. Luxembourg, Office for Official Publications of the European Communities, 2004.

International Atomic Energy Agency (IAEA). Radiation Protection and Safety of Radiation Sources: International Basic Safety Standards. IAEA Safety Standards for Protecting People and the Environment. General Safety Requirements Part 3. No. GSR Part 3. Vienna, International Atomic Energy Agency, 2014.

International Commission on Radiation Protection (ICRP). The 2007 Recommendations of the International Commission on Radiological Protection. ICRP Publication 103. Ann. ICRP, 37(24), 2007.

International Commission on Radiation Units and Measurements (ICRU). Patient dosimetry for $X$ rays used in medical imaging. Report 74. J. ICRU, 5(2):1-113, 2005.

Isaacson, K. G.; Thom, A. R.; Atack, N. E.; Horner, K. \& Whaites, E. Orthodontic Radiographs: Guidelines for the Use of Radiographs in Clinical Orthodontics. $4^{\text {th }}$ ed. London, British Orthodontic Society, 2015.
Ministerio de Salud - Instituto de Salud Pública. Manual de Protección Radiológica y de Buenas Prácticas en Radiología DentoMaxilo-Facial. Santiago de Chile, Ministerio de Salud, Instituto de Salud Pública, Gobierno de Chile, 2008. Disponible en: http:/ / w w w. m i n s a I. c I/ p o r t a I/ u rl/ i t e m/ 7f2d789a9750153be04001011f012d29.pdf

Miranda, P.; Ubeda, C.; Vaño, E. \& Nocetti, D. Recomendaciones para mejorar la seguridad radiológica durante los procedimientos de intervencionismo cardiológico. Rev. Chil. Cardiol., 33(1):44-50, 2014

Ruiz Manzano, P. Procedimientos Recomendados para la Dosimetría de Rayos de Energías entre 20 y 150 keV en Radiodiagnóstico. Madrid, Ramírez de Arellano Editores, Sociedad Española de Física Médica, 2005.

Ubeda, C. C.; Leyton, L. F.; Galaz, L. S.; Oyarzún, C. C. \& Inzulza, C. A. Garantía de calidad y protección radiológica en las exposiciones médicas en Europa. Un ejemplo a seguir. Rev. Chil. Radiol., 13(4):208-12, 2007.

Ubeda, C. C.; Nocetti, G. D.; Alarcón, E. R.; Inzulza, C. A.; Calcagno, Z. S.; Castro, B. M.; Vargas, C. J.; Leyton, L. F.; Oyarzún, C. C.; Ovalle, V. M. \& Torres, C. B. Magnitudes y unidades para dosimetría a pacientes en radiodiagnóstico e intervencionismo. Rev. Chil. Radiol., 21(3):94-9, 2015.

Ubeda, C.; Miranda, P.; Vaño, E. \& Nocetti, D. Protección radiológica en cardiología intervencionista pediátrica. Avances y desafíos para Chile. Rev. Chil. Cardiol., 32(3):223-9, 2013.

United Nations Scientific Committee on the Effects of Atomic Radiations (UNSCEAR). UNSCEAR 2008 Report: Sources and Effects of lonizing Radiation. Geneva, United Nations Scientific Committee on the Effects of Atomic Radiations (UNSCEAR), 2016. Disponible en: http://www.unscear.org/unscear/ publications/2008 1.html

Vandenberghe, B.; Jacobs, R. \& Bosmans, H. Modern dental imaging: a review of the current technology and clinical applications in dental practice. Eur. Radiol., 20(11):2637-55, 2010.

Vañó, E.; Miller, D. L.; Martin, C. J.; Rehani, M. M.; Kang, K.; Rosenstein, M.; Ortiz-López, P.; Mattsson, S.; Padovani, R.; Rogers, A. \& Authors on behalf of ICRP. ICRP Publication 135: Diagnostic Reference Levels in Medical Imaging. Ann. ICRP, 46(1):1-144, 2017.

Vañó, E.; Rosenstein, M.; Liniecki, J.; Rehani, M. M.; Martin, C. J. \& Vetter, R. J. ICRP Publication 113. Education and training in radiological protection for diagnostic and interventional procedures. Ann. ICRP, 39(5):7-68, 2009.

White, S. \& Pharoah, M. Oral Radiology: Principles and Interpretation. $7^{\text {th }}$ ed. St. Louis, Mosby, 2004.

Wold Dental Federation (FDI). FDI Policy Statement. Radiation Safety in Dentistry. New Delhi, FDI General Assembly, 2014. Disponible en: https://www.fdiworlddental.org/resources/policystatements-and-resolutions/radiation-safety-in-dentistry

Dirección para correspondencia:

Carlos Ubeda de la Cerda, MSc, PhD.

Decano Facultad de Ciencias de la Salud

Universidad de Tarapacá

Arica

CHILE

Email: cubeda@uta.cl

Recibido : 08-04-2018

Aceptado : 30-05-2018 